\title{
Antibody Exchange: Information Extraction of Biological Antibody Donation and a Web-Portal to Find Donors and Seekers
}

\author{
Sandeep Subramanian ${ }^{1}$ and Madhavi K. Ganapathiraju ${ }^{1,2, *}$ \\ 1 Language Technologies Institute, Carnegie Mellon University, Pittsburgh, PA 15213, USA; \\ sandeep.subramanian@gmail.com \\ 2 Department of Biomedical Informatics, and Intelligent Systems Program, University of Pittsburgh, \\ Pittsburgh, PA 15260, USA \\ * Correspondence: madhavi@pitt.edu or madhavicmu@gmail.com; Tel.: +1-412-624-5100
}

Received: 18 October 2017; Accepted: 17 November 2017; Published: 21 November 2017

\begin{abstract}
Bio-molecular reagents, like antibodies that are required in experimental biology are expensive and their effectiveness, among other things, is critical to the success of the experiment. Although such resources are sometimes donated by one investigator to another through personal communication between the two, there is no previous study to our knowledge on the extent of such donations, nor a central platform that directs resource seekers to donors. In this paper, we describe, to our knowledge, a first attempt at building a web-portal titled Antibody Exchange (or more general 'Bio-Resource Exchange') that attempts to bridge this gap between resource seekers and donors in the domain of experimental biology. Users on this portal can request for or donate antibodies, cell-lines, and DNA Constructs. This resource could also serve as a crowd-sourced database of resources for experimental biology. Further, we also studied the extent of antibody donations by mining the acknowledgement sections of scientific articles. Specifically, we extracted the name of the donor, his/her affiliation, and the name of the antibody for every donation by parsing the acknowledgements sections of articles. To extract annotations at this level, we adopted two approaches-a rule based algorithm and a bootstrapped pattern learning algorithm. The algorithms extracted donor names, affiliations, and antibody names with average accuracies of $57 \%$ and $62 \%$, respectively. We also created a dataset of 50 expert-annotated acknowledgements sections that will serve as a gold standard dataset to evaluate extraction algorithms in the future.
\end{abstract}

Data Set: Will be published as a supplement to this paper in the journal Data: http:/ /www.mdpi. com/2306-5729/2/4/38/s1.

Data Set License: CC-BY-NC-SA

Keywords: data exchange; resource donations; text mining

\section{Introduction}

Antibodies and other such wet-lab reagents are vital resources in a variety of experiments in molecular biology. These resources are expensive and their quality is crucial for the success of the experiment. For those conducting these experiments, it would be extremely valuable when these reagents become available in spare quantities in one lab, that they are then donated to others when required. This donation will be even more useful if the donor lab has already tested the quality of the resource. For example, a research group that studies the protein HMGB1 extensively, might have a reliable and well-tested antibody for it, and could potentially donate some of it to colleagues 
or collaborators who may need it. Such donations, where possible, could help unfunded junior investigators to carry out experiments that they otherwise could not afford. Further, such acts of generosity could spark collaborations between research groups and serve as a means to connect researchers with similar expertise.

Even in the strongly connected world that we are in today, researchers, unaware of a group that may have the reagents in close proximity within their organization, typically turn to vendors whose information is readily available online. Increasingly, there has been a trend towards open resource sharing. For example, open source software, open data sharing, and open access of manuscript publishing have become pervasive and have accelerated the advancement of science.

In these open sharing environments, what are the factors that drive people to do social good? While some individuals have altruistic motives, such as contributing to the advancement of science and encouraging junior investigators, there are others who build a reputation for being highly visible donors or build goodwill for future reciprocations. How feasible is it to share material resources among research groups, given that they cannot be shared simply over the Internet?

In this work, we studied the extent to which researchers share biological reagents, specifically antibodies, by parsing the acknowledgements sections of papers that are available in PubMed Central. Encouraged by what we found, we developed a web portal to connect donors with seekers of reagents to facilitate and promote the sharing of such resources. This portal can serve as a means for people to find locally available resources for their experiments.

The amount of bio-medically relevant content is increasing at an unprecedented rate; two new articles are published on PubMed every minute [1]. Therefore, information extraction from text documents has seen several advancements over the past decade [2-4]. The BioCreative and BioNLP workshop initiatives were created to evaluate text mining and information extraction approaches. Tasks ranging from named entity recognition (NER) on genes, drugs, and chemical compounds to protein-protein interaction extraction from PubMed have been a part of these initiatives $[5,6]$. Further, GENIA [7] is a dataset pertinent to text mining of bio literature and has played an important role in the advancement of Biomedical Natural Language Processing.

Riloff and Jones [8] pioneered an information extraction algorithm that iteratively learns rules for extracting relevant information and in turn uses the information to learn new rules. This approach to learning is often referred to as bootstrapping, and is in practice to-date [9-11]. Some of the biggest and most successful information extraction systems, like Never Ending Language Learner (NELL) [12], have used bootstrapping effectively even in the biomedical domain [13]. We adopt this as one of our methods to extract information from literature. The NLP research community has largely stuck to machine learning approaches for information extraction until very recently when rule based systems have seen some resurfacing, while the industry has always stuck to the latter [14]. Rule based information extraction systems have the advantage of being interpretable and can be fine-tuned easily [11]. In this work, we experiment with using a purely hand-engineered rule based extraction system and compare its performance with bootstrapped pattern learning system. Recently, bio-literature has been mined to index and curate bioinformatics and biomedical resources [15-17]. Several examples are presented in our prior work [18]. Duck et al. [19] present a literature mining approach to quantifying the use of resource in computational biology, while de la Calle et al. [17] do the same for resources in bioinformatics. In contrast, we focus on wet-lab resources. Ozyurt et al. [15] develop a holistic resource that has software, databases, and tissue banks, but does not contain antibodies or attempt to understand the phenomenon of bio resource donation in literature.

\section{Data Description and Methods}

Researchers acknowledge donations from others by thanking them in the acknowledgements section of their published work. In this particular work, we focused on studying acknowledgements pertaining to antibody donations. We mined full-text articles from PubMed Central to extract information at coarse and fine granularities. At the coarse level, we extracted the entire acknowledgement 
section if a case-insensitive search on the entire acknowledgement section contains the word "antibody" or "antibodies". Authors, however, tend to acknowledge multiple things in this section such as manuscript reading, instrument usage, and their funding sources. For example, the acknowledgement "We thank Peter Merrifield and Stefano Schiaffino for providing antibodies. This work was supported by grants from the Medical Research Council of Canada. K.E.M.H is a Killam Scholar of the Montreal Neurological Institute" contains information extraneous to the task that we are focused on. We therefore had to develop extraction algorithms that can carefully extract out donor names, donor affiliations, and antibody names from entire acknowledgement sections.

\subsection{Data Acquisition}

PubMed Central (PMC) provides full-text access to all of its open access papers https: / / www. ncbi.nlm.nih.gov/pmc/. As of April 2015, according to our statistics, it consisted of a total of $1,000,148$ open access papers. These papers are available for download, free of cost and formatted in XML. We parsed these to extract the acknowledgements section of every paper and searched for a reference to an antibody donation within it. Since generating the entire XML parse tree of every paper was computationally expensive, we ran a shallow parse using regular expressions to parse out just the acknowledgements section.

A crude extraction approach for this task was done using a case insensitive search for the word "antibody" and "antibodies" in the acknowledgements section, which returned 6533 instances across all of the papers in all of the journals. Only a very small fraction of these did not contain a reference to an antibody donation. For example, the sentence "We'd like to thank Doris Thelian for her expert advice on antibody cocktails and flow cytometry data analysis" has absolutely no reference to donation of an antibody.

We then analyzed the extracted paragraphs using information extraction algorithms that we will describe in detail in the subsequent sections to determine the antibody donor name, affiliation, and the name of the antibody.

\subsection{Rule Based Extraction}

Rule based systems can easily exploit the formal and consistent nature of writing in the acknowledgements section of scientific articles. Rule based information extraction systems that search key-word context windows have been employed with success in the past. The context in which a word occurs (i.e.), and the words surrounding it, has been studied extensively [20-23]. We formulate heuristically determined search rules for information extraction within these word contexts.

We observe that the antibody names were mostly written in "TARGET antibody" form (TARGET is the name of a protein or other biomolecule that the antibody binds to) (Figure 1), but also occasionally as "antibody of/to TARGET"; for example: "Dr. Y. Nishiyama is thanked for the antibody to UL7" is written in passive voice, which would require searching the right context instead. We assert that the first word within the left context window of the word "antibody" that is not in the English dictionary or a named entity, is the name of the antibody that was donated. If no such word is found in the left context, we then proceed to search the right-context. The size of the left and right context windows is set at four words, which are determined after examining the paragraphs from the high-level extraction step. Further, we also search the left context window for any tags (primary, secondary, monoclonal, polyclonal) that may associated with the antibody. While an NER system for antibodies would have been ideal, biomedical NER systems such as BANNER [24] are incapable of tagging antibodies nor is there a corpus from which a supervised one can be trained. 


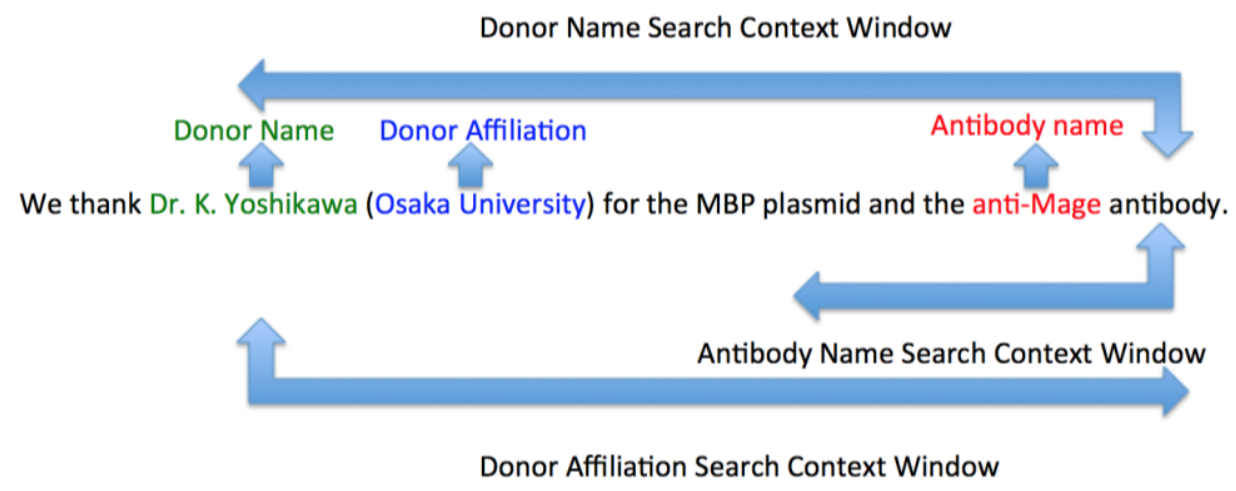

Figure 1. Rule based extraction.

While extracting the name of the donor, we do not fix the size of the context window in which we search. Instead, we keep searching the left context of the antibody name until a named entity that is labeled as a person is encountered. We found that the name of a donor is typically located far away from the antibody. We used MIT's Information Extraction Library (https://github.com/ mit-nlp/MITIE) (MITIE) for NER that identifies named entities and provides labels for them, like 'person', 'organization', and 'location'. Another observation about the nature of acknowledgements in biomedical literature was that a person's affiliation almost always occurred immediately after his/her name within brackets. We used this to label the donor's affiliation as the closest organization that was extracted by our NER in his/her right context, while still being on the left context of the word "antibody" or "antibodies".

\subsection{Bootstrapped Pattern Learning}

While rule-based extraction systems are capable of extracting entities with high precision, they require rules to be explicitly defined. This also prevents them from being easily adapted to new domains. Bootstrapping alleviates this problem by automatically learning phrases/patterns that identify entities of interest from seeded ground-truth annotations. The following subsection describes the bootstrapping algorithm that we used to learn extraction rules.

We used bootstrapping to identify antibody names only, using the context-based approach as described in the Rule Based Extraction to identify the donor names, affiliations, and antibody names. The algorithm is as follows:

- Seed an initial set of antibody names.

- For every sentence that contains any of the seeded antibody names, run a constituency parse to extract the leaves of immediate parent noun phrase, as shown in Figure 2, and replace the seeded antibody with a wildcard 'TARGET'.

- $\quad$ These phrases constitute the learned patterns. (Figure 3).

- Extract new antibody names using these learned patterns by pattern matching any of these patterns with every sentence.

- Repeat antibody-name extraction and rule-learning steps iteratively. 


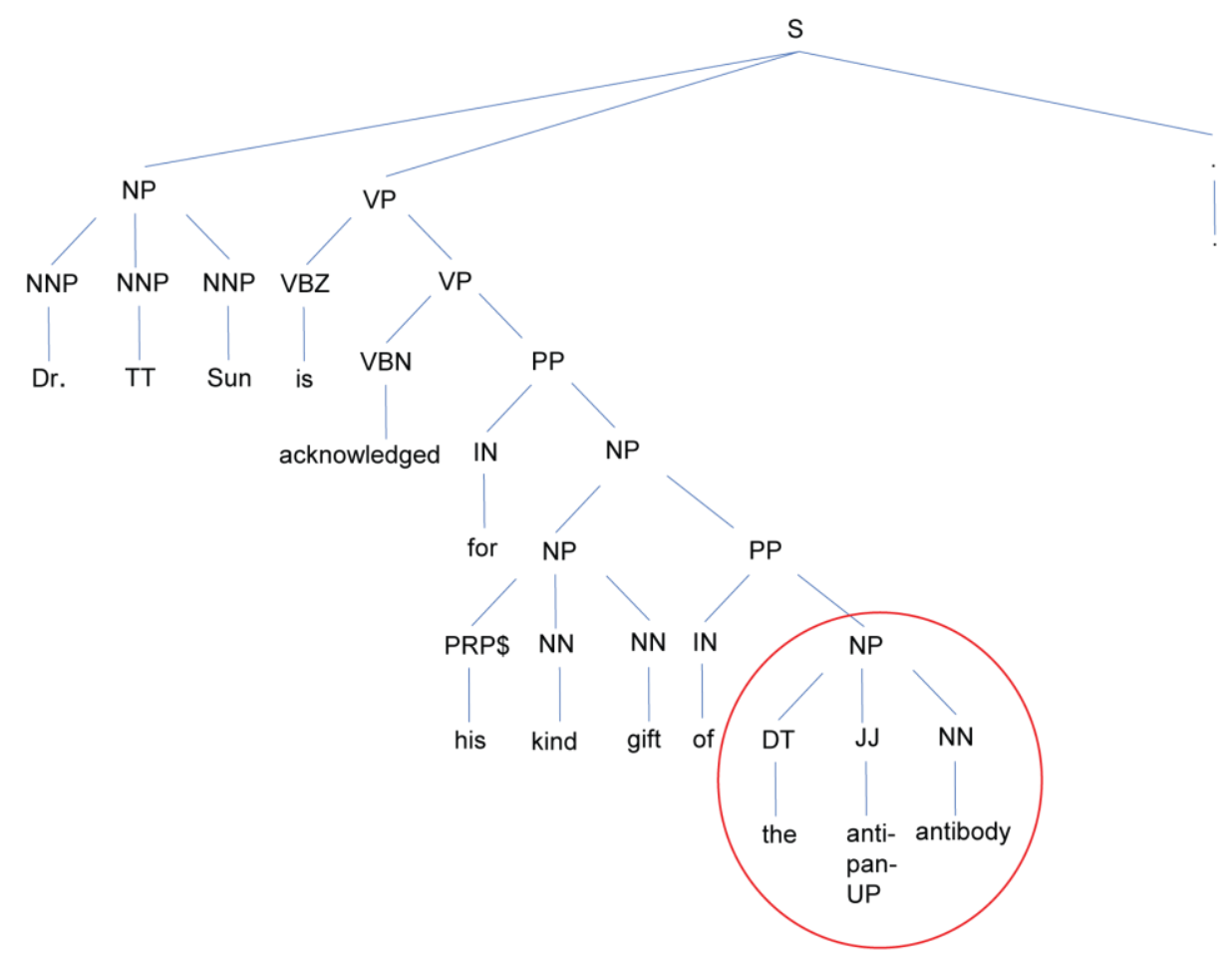

Figure 2. Constituency parse of a sentence to find an extraction rule.

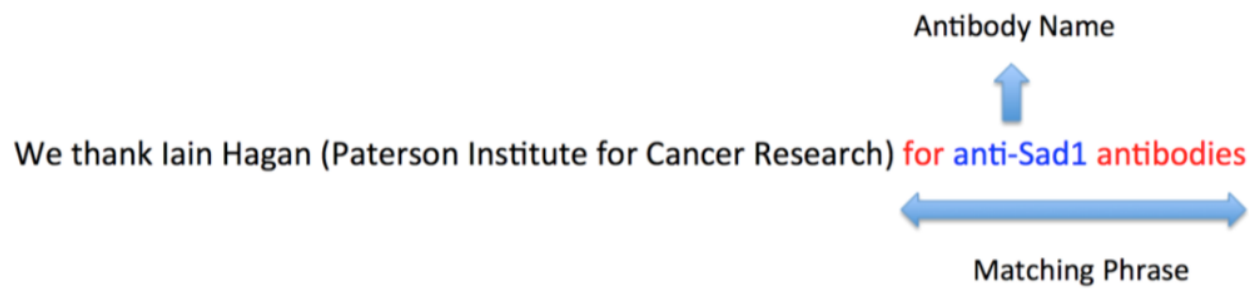

Matching Phrase : for ?? antibodies

Figure 3. Example extraction rule.

Bootstrapping algorithms learn new patterns and ground-truth iteratively $[2,25,26]$. The algorithm constitutes extracting antibody names either from the initial seed or from the patterns learned thus far, and then updating patterns from the current set of antibody names extracted. We observed that best performance was achieved after two iterations, whereas more iterations introduced noisy extraction rules.

Some of the patterns learned by this algorithm starting with 40 antibody names as seeds are:

- the mouse TARGET antibody

- rabbit TARGET antibodies

- TARGET monoclonal antibodies

- TARGET antibody

- $\quad$ antibody to TARGET

\subsection{Human Annotations}

There is no dataset with ground-truth annotations for evaluating these algorithms. So, we undertook the collection of human annotations for 50 randomly sampled acknowledgement sections. Biologists 
that are familiar with this domain were asked to manually annotate donor names, his/her affiliation, the name of the antibody, and any of its attributes. We also asked the annotators to identify other bio-resources (e.g., cell-lines) that they could find in the acknowledgements and annotate them with labels describing the resource and the resource name for future work along this line. Further, we also asked them to annotate people or organizations in the acknowledgements that were not a part of a donation of a bio-resource for potential application in NER tasks.

Example annotations of sentences describing only antibody donations are shown in Figures 4 and 5, and annotations of sentences that are containing other bio-resources are shown in Figure 6.

Person \& Donor

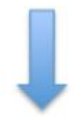

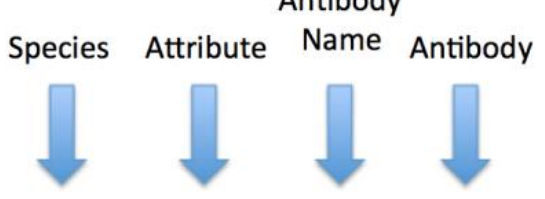

We are indebted to Dr C. Y. Chen for the generous gift of the mouse monoclonal anti-KSRP antibody

Figure 4. Example annotation of an antibody donation.

Person \& Donor

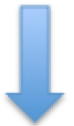

Organization \& Affiliation

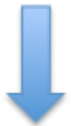

Antibody

Name Antibody

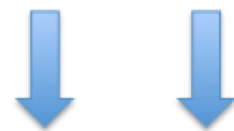

We thank lain Hagan (Paterson Institute for Cancer Research) for anti-Sad1 antibodies

Figure 5. Example annotation of an antibody donation.

\section{Organization \& Donor}

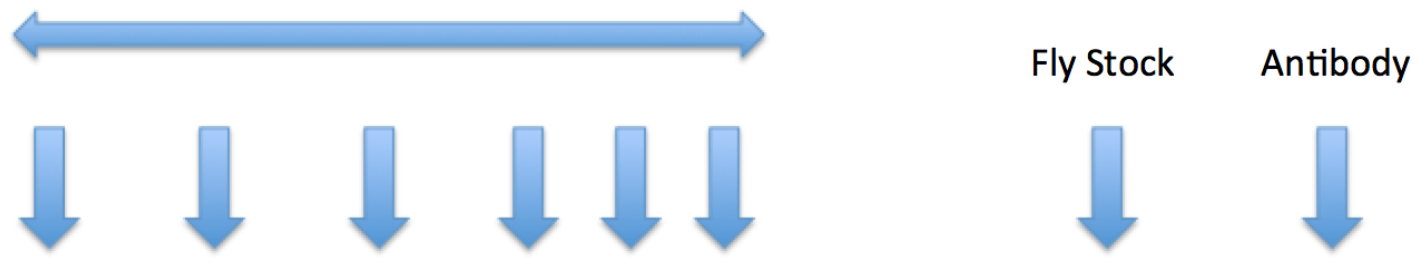

Bloomington, DSHB, BioServ UK, VDRC, NIG, DRSC are thanked for fly stocks and antibodies.

Figure 6. Example annotation of a fly stock donation.

Five biologists participated in the annotation process. Fifty abstracts were annotated overall, of which 18 were annotated by at least two individuals. Inter-annotator agreement was $75 \%$. We foresee these annotations being used as ground-truth for other researchers to evaluate their algorithms on the same task. More generally, these annotations could be used to train information extraction and named entity recognition systems. The annotations are formatted in XML, a snippet of which can be seen in Figure 7.

The dataset of annotated acknowledgements is provided in Supplementary Materials File 1. 


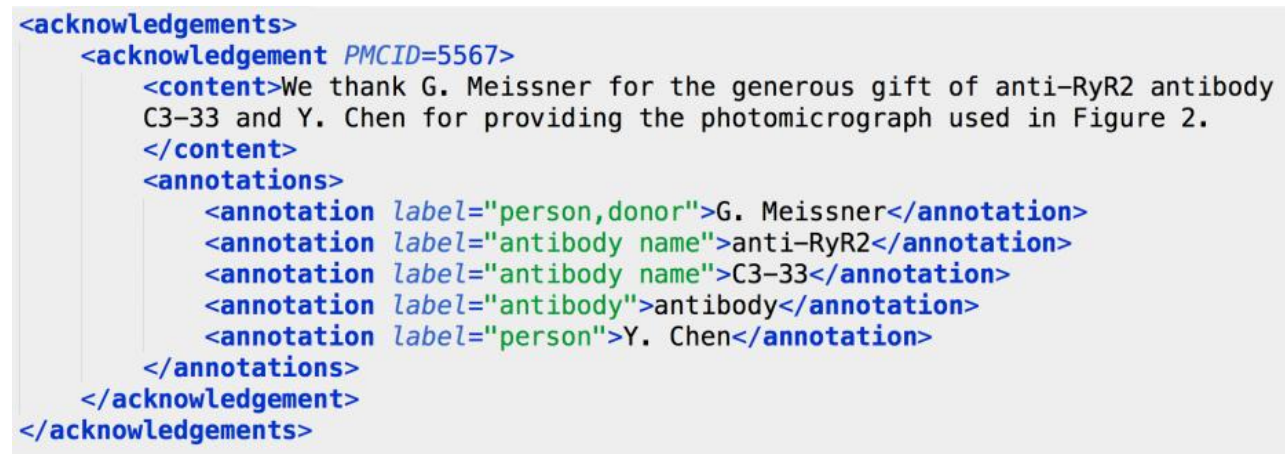

Figure 7. Human annotations formatted in XML.

\section{Results and Discussion}

We studied frequent donors (people \& organizations), frequently donated antibodies, and donation trends across journals, and trends over time. These results are presented for both approaches.

\subsection{Rule Based Approach}

The rule-based approach extracted a total of 7589 antibody donations. The number of extracted donations exceeds the number of acknowledgement sections because the algorithm is capable of extracting multiple donations within the same acknowledgement section. Table 1 contains the top five donor names irrespective of their affiliation.

Table 1. Top donors by name.

\begin{tabular}{cc}
\hline Donor & Number of Donations \\
\hline Keith Gull & 32 \\
Albert Einstein College of Medicine & 15 \\
Peter Davies & 12 \\
K. Gull & 10 \\
Hugo Bellen & 10 \\
\hline
\end{tabular}

Table 2 contains the top five donor-affiliation pairs.

Our approach suffers from some weaknesses—-the NER system tagged "Albert Einstein College of Medicine" as a person. Also, it is incapable of identifying different ways of writing a donor name (Keith Gull vs. K. Gull vs. Gull, K) or affiliation (University of Oxford vs. Oxford University).

Table 2. Top donors by donor-affiliation pairs.

\begin{tabular}{ccc}
\hline Donor & Affiliation & Number of Donations \\
\hline Keith Gull & University of Oxford & 6 \\
Keith Gull & Oxford University & 5 \\
Gary Ward & University of Vermont & 4 \\
K. Mackie & Indiana University & 3 \\
Yoshihiko Funae & Oosaka City University & 3 \\
\hline
\end{tabular}

Table 3 contains the organizations that donated the most antibodies. 
Table 3. Top donors by organization.

\begin{tabular}{cc}
\hline Donor & Number of Donations \\
\hline University of California & 24 \\
NIH & 19 \\
Rockefeller University & 15 \\
Harvard Medical School & 15 \\
University of Pennsylvania & 12 \\
\hline
\end{tabular}

Table 4 contains the most frequently donated antibodies.

Table 4. Most frequently donated antibodies.

\begin{tabular}{cc}
\hline Antibody Name & Number of Donations \\
\hline plasmids & 111 \\
autoantibody & 31 \\
DSHB & 28 \\
anti-tubulin & 14 \\
anti-GFP & 14 \\
\hline
\end{tabular}

Table 5 contains the journals that had the most references to an antibody donation. Note that these journals are completely open access, however all their articles are available in the data we processed.

Table 5. Journals with the most donations.

\begin{tabular}{cc}
\hline Journal & Number of Donations \\
\hline PLoS One & 2894 \\
PLoS Genetics & 667 \\
PLoS Pathology & 536 \\
PLoS ONE & 294 \\
PLoS Biology & 286 \\
\hline
\end{tabular}

Figure 8 shows a plot of the number of antibody donations extracted papers published during the years 2000 to 2014. The data the nature of open-access publications and their deposition in PubMed Central have seen increasing adoption during this period because of which the number of publications per year grew in PMC, from around 40 thousand in 2000 to about 400,000 thousand in 2014. To normalize for this effect, we also show 'donations per 1000 PMC articles', with a secondary axis on the right-side of the figure. We counted multiple donations that were mentioned in the same paper (of different antibodies or by different donors) as distinct entities; however, these are rare occasions and would not significantly alter normalized values. We can see that the donations themselves, or the practice of acknowledgement in the manuscripts, have increased over the years 2000-2014. 


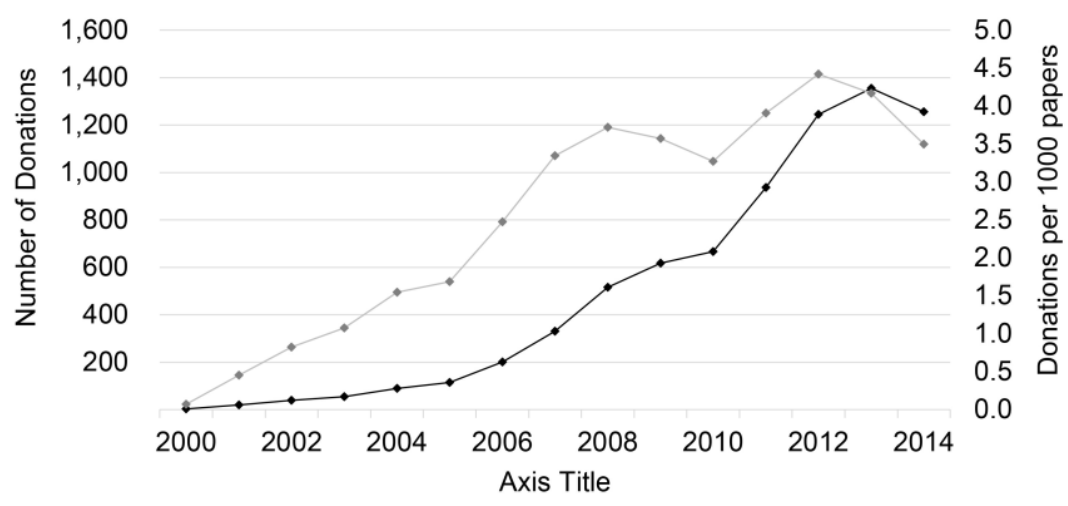

$\rightarrow$ Number of Donations $\rightarrow$ Avg Donations per 1000 Publications in PMC

Figure 8. Year vs. number of donations extracted in that year.

\subsection{Bootstrapped Pattern Learning}

The bootstrapped pattern learning algorithm extracted a total of 7864 antibody donations. Table 6 contains the top five donors independent of their affiliation.

Table 6. Top donors by name.

\begin{tabular}{cc}
\hline Donor & Number of Donations \\
\hline Keith Gull & 24 \\
Albert Einstein College of Medicine & 20 \\
Erich Buchner & 11 \\
Charles Rice & 11 \\
K. Gull & 10
\end{tabular}

Table 7 contains the top five donor-affiliation pairs.

Table 7. Top donors by donor-affiliation pair.

\begin{tabular}{ccc}
\hline Donor & Affiliation & Number of Donations \\
\hline Dr. Charles Rice & Rockefeller University & 9 \\
Steven S. Gross & Weill Medical College & 8 \\
Harold Gainer & NIH & 7 \\
Keith Gull & University of Oxford & 7 \\
Gary Ward & University of Vermont & 6 \\
\hline
\end{tabular}

Table 8 contains the organizations that donated the most antibodies.

Table 8. Top donors by organization.

\begin{tabular}{cc}
\hline Donor & Number of Donations \\
\hline NIH & 23 \\
Harvard Medical School & 22 \\
Rockefeller University & 21 \\
University of California & 20 \\
University of Pennsylvania & 20 \\
\hline
\end{tabular}

Table 9 below contains the antibody names that were donated the most frequently. 
Table 9. Most frequently donated antibodies.

\begin{tabular}{cc}
\hline Antibody Name & Number of Donations \\
\hline plasmids & 74 \\
anti-mouse & 30 \\
anti-gfp & 18 \\
anti-tubulin & 12 \\
anti-actin & 10 \\
\hline
\end{tabular}

Table 10 contains the journals that had the most references to an antibody donation.

Table 10. Most frequently donated antibodies.

\begin{tabular}{cc}
\hline Journal & Number of Donations \\
\hline PLoS One & 3174 \\
PLoS Pathology & 671 \\
PLoS Genetics & 577 \\
PLoS Biology & 306 \\
PLoS ONE & 301 \\
\hline
\end{tabular}

The plot showing the temporal donation trend for this approach was identical to the previous approach and is therefore not included in this section.

\section{Extraction Evaluation}

\section{Evaluation}

We evaluated the performance of our algorithms, by comparing them to any of the human labeled annotations. We report the accuracies in Table 11. It is evident that we are able to extract characteristics about an antibody using both our proposed approaches. The bootstrapped pattern learning algorithm achieves a better performance than the simple rule-based approach at extracting donor and antibody names but does not do as well with extracting affiliations.

Table 11. Extraction results.

\begin{tabular}{ccccc}
\hline \multirow{2}{*}{ Approach } & \multicolumn{3}{c}{ Accuracy } \\
\cline { 2 - 5 } & Donor & Affiliation & Antibody Name & Mean \\
\hline Rule Based & $50 \%$ & $70 \%$ & $50 \%$ & $57 \%$ \\
Bootstrapped Pattern Learning & $57 \%$ & $66 \%$ & $64 \%$ & $62 \%$ \\
\hline
\end{tabular}

\section{Bio-Resource Exchange Web Portal}

We developed a resource-sharing web-portal, called Bio-Resource Exchange (BRX) available at http:/ / tonks.dbmi.pitt.edu/brx. It is built modularly, with the ability to be a generic resource-sharing platform. It allows for users to make requests for or donate resources via a simple customizable form for each resource. At present, resources on BRX include antibodies, DNA constructs, and cell-lines. The moment that a form is filled in by a user, it appears on a bulletin board (analogous to a news feed on social networking websites) that is visible to all of the other users in the system (Figure 9). A user's news feed may be filtered based on the type of research he/she is looking for. It allows users to search for specific information, for example, particular antibodies or cell-lines, or posts by specific individuals. BRX also allows users to comment on posts and also puts them in touch with the author via email. 


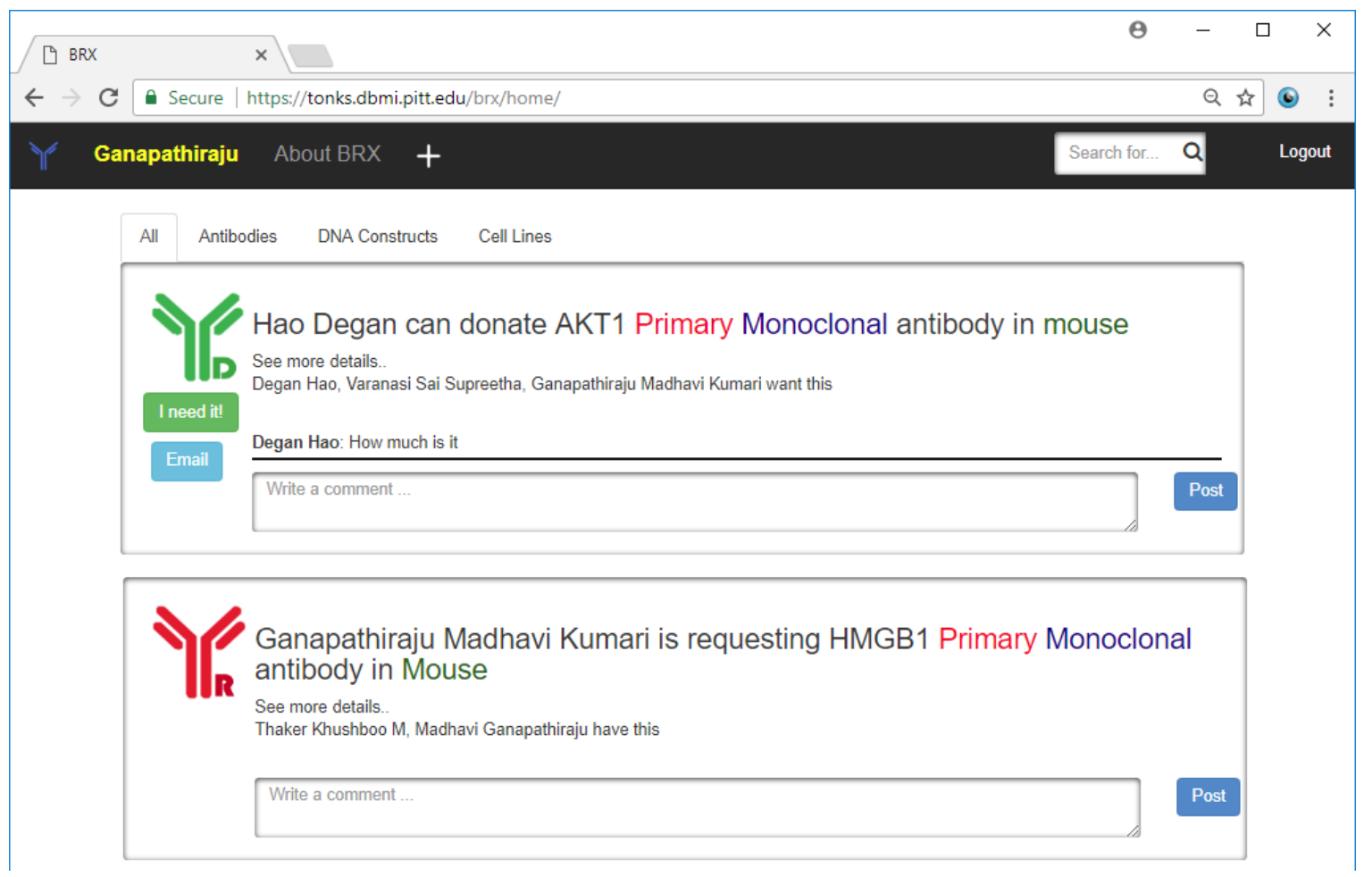

Figure 9. Screenshot of the Web-Portal.

BRX was developed using the Django web framework with a MySQL backend database. Separate tables were created for each resource type to allow for each of them to have different attributes using Django's ORM (Object-relation Mapping). Foreign keys to this table were made to store comments and email correspondences. The rest of the backend elements are designed to make the addition of a new resource extremely simple. Third-party authentication elements on BRX were built using an open source Oauth2 library (https:/ / github.com/omab/python-social-auth). For University of Pittsburgh users exclusively, we used LDAP (Lightweight Directory Access Protocol) authentication to let users sign in with their university email accounts. Front-end elements were built using twitter-bootstrap, Vanilla JavaScript, and jQuery. In the future, the front-end could include leaderboards of universities, organizations, and people who have donated the most resources to promote healthy competition.

The results from mining literature have not been incorporated into the website, because the posts are tied to individual profiles, i.e., of donors and seekers. Unless a user registers and posts the information that they are seeking/donating antibodies or other resources, it does not appear on the web portal.

\section{Conclusions}

We carried out text-mining over acknowledgement sections of open-access articles to study the extent of antibody donations reported so far. We first created a dataset of 50 expert-annotated acknowledgements sections that will be useful for algorithm development and evaluation purposes in studying such donations. Using NLP techniques, we extracted the name of the donor, his/her affiliation, and the name of the antibody for every donation by parsing the sentences by adopting two approaches-a rule based algorithm and a bootstrapped pattern learning algorithm, and achieved average accuracies of $57 \%$ and $62 \%$, respectively.

We also developed a web-portal, Bio-Resource Exchange, which attempts to connect biologists seeking antibodies, cell-lines, or DNA-constructs to potential donors. We expect that it would bridge a gap between resource seekers and donors in the domain of experimental biology. Users on this portal 
can post information that they are either seeking a specific antibody, cell-line, or DNA construct, or that they are in a position to donate them. It allows for other registered users to comment on such posts. Thus, this resource could grow into a crowd-sourced database of resources for experimental biology.

\section{Limitations}

A significant limitation of this work is that the text-mining methods that were adopted were extremely simple and involved the use of several heuristics owing to limited data and the absence of a labeled corpus for such a task. There exist no corpora from which named entity taggers can be learned to recognized antibody names in a supervised manner, and therefore more contemporary methods, such as CRFs [27] or neural methods [28], could not be adopted. While integrating results from text-mining is trivial from an implementation perspective, some thought has to be put into curating the data that goes onto the web page. For example, attributing an antibody with an incorrect donor could lead to problems when contacting him/her. Further, we found it difficult to get biologists to annotate a large collection of our dataset and so had to distribute our data across many of them, thereby inducing a minimal amount of noise in the annotations. Soft matching constraints when evaluating our models could provide deeper insights into the model's strengths and weaknesses. At present, the XML dataset does not contain the character offsets of each annotation within the paragraph - this is an extension that we foresee adding in future releases of this dataset.

Supplementary Materials: Supplementary materials can be found at http:/ /www.mdpi.com/2306-5729/2/4/ 38/s1.

Acknowledgments: We thank Vishwajit L. Nimgaonkar and researchers in his group Chowdari Kodavali, Lora McClain, Joel Wood, Tulsi Malavia, and Srilakshmi Chaparala for annotating the sentences we collected. We thank Srilakshmi Chaparala and Adam Handen for also providing valuable inputs on the Bio-Resource Exchanged website. We thank Lucy Cafeo for reading the revised version of the manuscript and providing language edits. This work is funded in part by the BRAINS grant R01MH094564 from the National Institute of Mental Health of the National Institutes of Health (NIMH/NIH), USA. We thank the reviewers for their comments which helped improve the paper.

Author Contributions: M.K.G. conceptualized and supervised the work, and designed the web-portal. S.S. proposed and implemented data acquisition and text mining algorithms, and developed the web portal. Article has been written, edited and approved by both the authors.

Conflicts of Interest: The authors declare no conflict of interest.

\section{References}

1. Nawaz, R.; Thompson, P.; Ananiadou, S. Negated bio-events: Analysis and identification. BMC Bioinform. 2013, 14, 14. [CrossRef] [PubMed]

2. Finkel, J.R.; Grenager, T.; Manning, C. Incorporating non-local information into information extraction systems by gibbs sampling. In Proceedings of the 43rd Annual Meeting on Association for Computational Linguistics, Ann Arbor, MI, USA, 25-30 June 2005; pp. 363-370.

3. Banko, M.; Cafarella, M.J.; Soderland, S.; Broadhead, M.; Etzioni, O. Open information extraction for the web. In Proceedings of the IJCAI, Hyderabad, India, 6-12 January 2007; pp. 2670-2676.

4. Soderland, S. Learning information extraction rules for semi-structured and free text. Mach. Learn. 1999, 34, 233-272. [CrossRef]

5. Hirschman, L.; Yeh, A.; Blaschke, C.; Valencia, A. Overview of BioCreAtIvE: Critical assessment of information extraction for biology. BMC Bioinform. 2005, 6, S1. [CrossRef] [PubMed]

6. Kim, J.-D.; Pyysalo, S.; Ohta, T.; Bossy, R.; Nguyen, N.; Tsujii, J.I. Overview of BioNLP shared task 2011. In Proceedings of the BioNLP Shared Task 2011 Workshop, Portland, OR, USA, 24 June 2011; pp. 1-6.

7. Kim, J.-D.; Ohta, T.; Tateisi, Y.; Tsujii, J.I. GENIA corpus-A semantically annotated corpus for bio-textmining. Bioinformatics 2003, 19, i180-i182. [CrossRef] [PubMed]

8. Riloff, E.; Jones, R. Learning dictionaries for information extraction by multi-level bootstrapping. In Proceedings of the AAAI/IAAI, Orlando, FL, USA, 18-22 July 1999; pp. 474-479.

9. Dempster, A.P.; Laird, N.M.; Rubin, D.B. Maximum likelihood from incomplete data via the EM algorithm. J. R. Stat. Soc. Ser. B Stat. Methodol. 1977, 39, 1-38. 
10. Gupta, S.; Manning, C.D. SPIED: Stanford Pattern-Based Information Extraction and Diagnostics. In Proceedings of the ACL 2014 Workshop on Interactive Language Learning, Visualization, and Interfaces (ACL-ILLVI), San Francisco, CA, USA, 27 June 2014; p. 38.

11. Gupta, S.; Manning, C.D. Improved Pattern Learning for Bootstrapped Entity Extraction. CoNLL-2014 2014, 98. [CrossRef]

12. Carlson, A.; Betteridge, J.; Kisiel, B.; Settles, B.; Hruschka, E.R., Jr.; Mitchell, T.M. Toward an Architecture for Never-Ending Language Learning. In Proceedings of the AAAI, Atlanta, GA, USA, 11-15 July 2010; p. 3.

13. Movshovitz-Attias, D.; Cohen, W.W. Bootstrapping biomedical ontologies for scientific text using nell. In Proceedings of the 2012 Workshop on Biomedical Natural Language Processing, Montreal, QC, Canada, 3-8 June 2012; pp. 11-19.

14. Chiticariu, L.; Li, Y.; Reiss, F.R. Rule-Based Information Extraction is Dead! Long Live Rule-Based Information Extraction Systems! In Proceedings of the EMNLP, Seattle, WA, USA, 18-21 October 2013; pp. 827-832.

15. Ozyurt, I.B.; Grethe, J.S.; Martone, M.E.; Bandrowski, A.E. Resource Disambiguator for the Web: Extracting Biomedical Resources and Their Citations from the Scientific Literature. PLoS ONE 2016, 11, e0146300. [CrossRef] [PubMed]

16. Petersen, R.; Kempler, G.; Barklis, E. A stem cell-specific silencer in the primer-binding site of a retrovirus. Mol. Cell. Biol. 1991, 11, 1214-1221. [CrossRef] [PubMed]

17. de la Calle, G.; Garcia-Remesal, M.; Chiesa, S.; de la Iglesia, D.; Maojo, V. BIRI: A new approach for automatically discovering and indexing available public bioinformatics resources from the literature. BMC Bioinform. 2009, 10, 320. [CrossRef] [PubMed]

18. Roth, A.; Subramanian, S.; Ganapathiraju, M.K. Towards extracting supporting information about predicted protein-protein interactions. IEEE/ACM Trans. Comput. Biol. Bioinform. 2015. [CrossRef] [PubMed]

19. Duck, G.; Nenadic, G.; Brass, A.; Robertson, D.L.; Stevens, R. bioNerDS: Exploring bioinformatics' database and software use through literature mining. BMC Bioinform. 2013, 14, 194. [CrossRef] [PubMed]

20. Mikolov, T.; Sutskever, I.; Chen, K.; Corrado, G.S.; Dean, J. Distributed representations of words and phrases and their compositionality. In Proceedings of the Advances in Neural Information Processing Systems, Lake Tahoe, NV, USA, 5-10 December 2013; pp. 3111-3119.

21. Pennington, J.; Socher, R.; Manning, C.D. Glove: Global vectors for word representation. In Proceedings of the Empiricial Methods in Natural Language Processing (EMNLP 2014), Doha, Qatar, 25-29 October 2014; Volume 12, pp. 1532-1543.

22. Turian, J.; Ratinov, L.; Bengio, Y. Word representations: A simple and general method for semi-supervised learning. In Proceedings of the 48th Annual Meeting of the Association for Computational Linguistics, Uppsala, Sweden, 11-16 July 2010; pp. 384-394.

23. Tang, B.; Cao, H.; Wang, X.; Chen, Q.; Xu, H. Evaluating word representation features in biomedical named entity recognition tasks. BioMed Res. Int. 2014, 2014, 240403. [CrossRef] [PubMed]

24. Leaman, R.; Gonzalez, G. BANNER: An executable survey of advances in biomedical named entity recognition. In Proceedings of the Pacific Symposium on Biocomputing, Kohala Coast, HI, USA, 4-8 January 2008; pp. 652-663.

25. Wu, X.; Fan, J.; Subramanian, K.R. B-EM: A classifier incorporating bootstrap with em approach for data mining. In Proceedings of the Eighth ACM SIGKDD International Conference on Knowledge Discovery and Data Mining, Edmonton, AB, Canada, 23-26 July 2002; pp. 670-675.

26. McCallumz, A.; Nigam, K. Text Classification by Bootstrapping with Keywords, EM and Shrinkage. In Proceedings of the Workshop for Unsupervised Learning in Natural Language Processing, Ann Arbor, MI, USA, 21 June 1999.

27. Settles, B. Biomedical named entity recognition using conditional random fields and rich feature sets. In Proceedings of the International Joint Workshop on Natural Language Processing in Biomedicine and its Applications, Geneva, Switzerland, 28-29 August 2004; pp. 104-107.

28. Lample, G.; Ballesteros, M.; Subramanian, S.; Kawakami, K.; Dyer, C. Neural architectures for named entity recognition. arXiv, 2016.

(C) 2017 by the authors. Licensee MDPI, Basel, Switzerland. This article is an open access article distributed under the terms and conditions of the Creative Commons Attribution (CC BY) license (http:/ / creativecommons.org/licenses/by/4.0/). 